\title{
Políticas públicas para bibliote- cas públicas: una propuesta de soluciones locales a problemas globales *
}

\author{
Orlanda Jaramillo \\ Didier Álvarez Zapata \\ Daniel Moncada P. **
}

Artículo recibido:

19 de octubre de 2004.

Artículo aceptado:

7 de enero de 2005.

\section{RESUMEN}

Como parte del proyecto de investigación "Pautas para la implementación de políticas públicas para las bibliotecas públicas de los municipios de Antioquia”, el artículo considera a la biblioteca pública como una institución social y política promotora del desarrollo social y de la participación ciudadana. Esta noción, se dice, necesita hacerse plenamente visible ante el Estado y la sociedad, y frente a las instituciones realacionadas con estas bibliotecas, como marcos que consideren la función de la biblioteca pública dentro de determinados

* Las consideraciones del artículo, hacen parte del marco referencial de la investigación "Pautas para el diseño e implementación de políticas públicas para las bibliotecas públicas de los municipios de Antioquia", desarrollada por el Grupo de Investigación en Biblioteca Pública.

** Los tres autores pertenecen a la Escuela Interamericana de Bibliotecología, Universidad de Antioquia, Colombia. (Orlanda: ojara@nutabe.udea.edu.co); (Didier: dial@nutabe.udea.edu.co); (Daniel: eibpublicas@nutabe.udea.edu.co). 
contextos y proyectos sociales. Más específicamente, presenta reflexiones, antecedentes y postulados para formular e implementar políticas públicas destinadas a las bibliotecas públicas en el contexto colombiano actual. Para ello reflexiona en torno a la necesidad y conveniencia de crear políticas relacionadas con instituciones tomando en cuenta su contexto y entorno.

Palabras clave: Biblioteca pública; Políticas públicas; Desarrollo local; Desarrollo social; Participación ciudadana.

\section{ABSTRACT \\ Public policies for public libraries: a proposal for lo- cal solutions to global problems \\ Orlanda-Jaramillo; Didier Álvarez-Zapata and \\ Daniel-Moncada}

As part of the research project "Guidelines for the implementation of public policies for public libraries of Antioquia's town councils", this paper considers the public library as a social and political institution devoted to social development and citizen participation. This notion needs to become fully evident to the State and Society, and also to the institutions dealing with public libraries, as frame which considers the function of such libraries in their true context and as social projects. More specifically it reflects on basic principles in order to formulate and implement public action for these type of libraries in the present Colombian context.

Keywords: Public library; Public policies; Local development; Social development; Citizen participation.

\section{INTRODUCCIÓN}

E ste artículo se mueve dentro del campo de reflexión de lo político, un campo, por cierto, comúnmente ajeno a la reflexión bibliotecológica pero definitivo para las prácticas bibliotecarias, especialmente para las de la biblioteca pública. Y lo hace abordando un tema que viene emergiendo con 
mucha fuerza en los últimos años en el pensamiento y la práctica social latinoamericana: la formulación de políticas públicas. Particularmente, el artículo explora las formas y posibilidades de desarrollar una política pública para las bibliotecas públicas de Colombia. Para ello se parte de la consideración de que el conjunto de relaciones entre biblioteca pública, sociedad y Estado en este país, presenta un especial interés no sólo por los agudos problemas sociales que se han vivido en los últimos cincuenta años, sino también por las particulares condiciones y características de desarrollo que ha tenido la biblioteca pública en tal desarrollo. De hecho, en Colombia estas relaciones se dieron durante la segunda mitad del siglo XX a través de modelos atípicos (en comparación con los seguidos por la biblioteca en otras regiones de América Latina), que se expresaron, por ejemplo, en la creación de bibliotecas públicas atendidas y dirigidas por el sector privado a través, principalmente, de las cajas de compensación familiar, y en el trabajo educativo y organizativo de las comunidades urbanas emprendido en las grandes ciudades colombianas por las bibliotecas populares.

En este contexto, la biblioteca pública se ha inscrito, en no pocos casos, en el desarrollo de un proyecto democrático que se declara multicultural, de apoyo a la educación y que va de la mano de la globalización cultural, pero sin plantearse una reflexión política que la lleve al cumplimiento de sus objetivos de democratización de la información, la cultura y la recreación para todos. Desde esta perspectiva, la biblioteca pública no logra aún inscribirse en un marco amplio que le permita formar parte de los programas de desarrollo educativo y cultural del gobierno.

En este desarrollo a la colombiana de la biblioteca pública, debe anotarse, persisten dificultades para representar a la biblioteca como una institución que se debe a la tarea de favorecer "espacios de emancipación de los sujetos en la sociedad"; ${ }^{1}$ esto es, a reconocerla como una institución con responsabilidad política que haga efectivo el mandato constitucional de

garantizar a toda persona la libertad de expresar y difundir su pensamiento y opiniones, la de informar y recibir información veraz e imparcial, y la de fundar medios masivos de información. ${ }^{2}$

Esta dificultad se traduce en la poca consideración que hacen las bibliotecas del derecho a la información como una acción social que fomente una

1 Didier Álvarez Zapata. "El papel democratizador de la biblioteca pública popular: Retos y perspectivas políticas", en Memorias del Encuentro de Bibliotecarios Populares: Por el Fortalecimiento de las Dinámicas Sociales. Medellín : Biblioteca Pública Piloto, 2002. p. 22.

2 COlombia. Congreso. Constitución Política de Colombia 1991. Bogotá : El Congreso, 1991. p. 15. 
ciudadanía responsable y fundada sobre el pleno reconocimiento de los derechos humanos, y el contexto del país y la región.

Pero las dificultades no solamente se encuentran en la esfera de la comprensión teórica sobre la biblioteca pública y sus objetivos, también se reflejan en un componente esencial del problema que aborda la investigación que dio origen a este artículo: la muy insuficiente integración de la biblioteca pública en las políticas públicas de carácter estatal y gubernamental; políticas que articulen efectivamente los planes, programas, proyectos y demás acciones encaminadas a desarrollar la biblioteca pública y a insertarla en la vida política de las personas.

Es en este sentido en el que el tema de políticas públicas sobre bibliotecas públicas (asumidas como "programas de acción gubernamental, en un sector de la sociedad y en un espacio geográfico", ${ }^{3}$ con claras pretensiones de cambio social) se hace indispensable para discutir los futuros de la sociedad colombiana; es decir, para concertar a la sociedad civil y al Estado alrededor del valor, el lugar y las tareas que tiene la biblioteca pública en los propósitos para democratizar cultural, social, económica y políticamente al país. De esta forma aportará estrategias para promover y encauzar las demandas y las necesidades de la base social, e impulsará las necesarias transformaciones del Estado.

En consecuencia este artículo tiene como objetivo iniciar una reflexión en torno a la necesidad y conveniencia de desarrollar políticas públicas para las bibliotecas públicas en Colombia, que estén acordes con el contexto local y con la promoción de la participación ciudadana. Con este fin inicia considerando algunos antecedentes generales sobre la formulación de las políticas públicas, continúa con los postulados para su formulación, hace luego algunas consideraciones metodológicas previas, y termina planteando propuestas para su adopción e implementación.

\section{Antecedentes generales}

Es común que a la biblioteca pública en América Latina se la quiera representar como una institución democrática casi exclusivamente por el hecho de ser pública, pero esta consideración olvida que la promoción y el acceso a los bienes culturales que permite la biblioteca debe estar acompañada de acciones relacionadas con su reivindicación social y política. Concretamente es bien conocido que diversos organismos han elaborado directrices que plantean un

3 Bruno Revesz. "Políticas públicas en América Latina y el papel estratégico de las bibliotecas públicas: nuevos desafíos”, en Reunión Regional de Bibliotecas Públicas. Piura: IFLA, 1996. p. 3. 
marco conceptual, legal y administrativo para la biblioteca pública. Tal marco se plasma en documentos que tienen un creciente valor indicativo y un gran poder simbólico, además de que orientan la misión, el funcionamiento y la gestión de la biblioteca pública.

Tales documentos son, principalmente, los Manifiestos UNESCO para la Biblioteca Pública ${ }^{4}$ (en su tres versiones correspondientes a los años 1949, 1972 y 1994) y las Directrices y Pautas IFLA $^{5}$ (de los años 1972, 1985 y 2001). Estos documentos tienen su correlato, en lo que respecta a América Latina, en la ya lejana Declaración de Caracas sobre la Biblioteca Pública como Factor de Desarrollo e Instrumento de Cambio en América Latina y El Caribe que plantea el marco conceptual y el devenir de la biblioteca pública para la región, reafirmando el papel del Estado en cuanto a su funcionamiento y desarrollo, y enfatizando la necesidad de que las bibliotecas sean incluidas en los planes de desarrollo de cada país, al señalar la "obligatoriedad del Estado para ofrecer los servicios bibliotecarios públicos y asignarle a su desarrollo, anualmente, partidas presupuestales suficientes". ${ }^{7}$

Pero esta representación de la biblioteca exige reflexionar sobre la institucionalidad bibliotecaria pública desde una perspectiva política y no sólo desde lo administrativo, de forma que se haga claro y viable en los discursos y las prácticas bibliotecarias reclamar para ella una coalición de fuerzas sociales y estatales dirigida a hacerla visible como elemento crucial del desarrollo social. Actitud que en el caso de Colombia no ha sido desarrollada y que ha privado al país de una política pública sobre biblioteca pública. Cuando mucho podríamos hablar de una política nacional de información poco clara y de ciertas menciones más bien desdibujadas sobre la biblioteca pública en las políticas de educación y cultura que se han producido en los últimos veinte años en Colombia, en especial, a la luz de la nueva constitución de 1991.

4 La UNESCO interviene en el desarrollo de las biblioteca públicas con la publicación del "Manifiesto Sobre la Biblioteca Pública" en 1949, 1972 y 1994, documentos que han sido guía metodológica e ideológica en la creación y el desarrollo de las bibliotecas públicas en el mundo y, específicamente, en Colombia. Concretamente, por ejemplo, los planteamientos del Manifiesto de 1949, sustentaron la creación de la Biblioteca Pública Piloto de Medellín para América Latina.

5 La IFLA, Federación Internacional de Asociaciones e Instituciones Bibliotecarias, en unión con la UNESCO, publica en el año 2000 la tercera versión de las Directrices IFLA/UNESCO para el Desarrollo de Servicios de Bibliotecas Públicas. Documento que plantea lineamientos para la implementación y desarrollo de la biblioteca pública.

6 Documento elaborado en el marco de la Reunión Sobre el Estado Actual y Estrategias para el Desarrollo de las Bibliotecas Públicas en América Latina y el Caribe, celebrada entre diversos expertos en biblioteca pública de países Latinoamericanos.

7 REUNIÓN REGIONAL SOBRE EL ESTADO ACTUAL Y LAS ESTRATEGIAS PARA EL DESARROLLO DE LOS SERVICIOS DE BIBLIOTECAS PÚbLICAS EN AMÉRICA LATINA Y EL CARIBE. "Declaración de Caracas sobre la biblioteca pública como factor de desarrollo e instrumento de cambio social en América Latina y el Caribe”, en Bibliotecas públicas y escolares. Bogotá: Fundalectura, 2001. p. 175. 
En la recuperación de los rasgos arriba mencionados del discurso estatal sobre la biblioteca pública, habría que mencionar, más particularmente, que en los años 70 se sentaron las bases del Sistema Nacional de Información, y que como estrategia de apoyo a éste se crearon diversos institutos del orden nacional y de carácter descentralizado, entre ellos COLCULTURA, a partir del cual se impulsaron políticas relacionadas con la información y la cultura. De hecho, los programas y acciones impulsados y realizados desde COLCULTURA animaron la circulación de un discurso normativo propicio a las bibliotecas públicas y la promulgación de algunas leyes, decretos, acuerdos y otras disposiciones legales aisladas de las cuales se pueden inferir asomos de una consideración política sobre las bibliotecas, el libro, la lectura y los actores sociales involucrados en el proceso de transferencia de la información y el conocimiento: autores, lectores, usuarios, editores y libreros.

Un buen ejemplo de ello es la creación en 1973 del Sistema Nacional de Información en Ciencia y Tecnología (adscrito al Instituto Colombiano para el Desarrollo de la Investigación, la Ciencia y la Tecnología —COLCIENCIAS) ${ }^{8}$ que se planteó el objetivo de

poner a disposición de toda la comunidad nacional los recursos de información existentes en el país, para lo cual debe coordinar las acciones y los recursos necesarios y estructurar una red nacional de bibliotecas y centros de información. ${ }^{9}$

Precisamente en 1999, COLCIENCIAS presenta en la Conferencia General de la IFLA, ${ }^{10}$ la ponencia: Políticas Nacionales de Información $;{ }^{11}$ trabajo que recoge los avances en la elaboración e implementación de una Política Nacional de Información, desde las primeras acciones realizadas en los años 60. El documento, presenta fundamentalmente una propuesta metodológica para construir e implementar la política, para lo cual inicia con la descripción de conceptos básicos; continúa con la formulación que comprende la evaluación del entorno, la preparación de documentos, la consulta nacional y la escritura del proyecto; luego plantea las estrategias para su ejecución

8 COLCIENCIAS: Instituto para el Desarrollo de la Investigación "Francisco José de Caldas" es el ente encargado del fomento y desarrollo de las políticas nacionales de información en Colombia.

9 COLCIENCIAS. Sistema Nacional de Ciencia y Tecnología: instrumentos jurídicos. Bogotá: COLCIENCIAS, 1988. p. 15.

10 La Federación Internacional de Asociaciones Bibliotecarias, IFLA, realiza anualmente la "Conferencia General", uno de los principales eventos en el ámbito de la bibliotecología en el que se presentan los trabajos más relevantes en este campo realizados en todos los países del mundo. La conferencia de 1999, realizada en Bangkok (Tailandia), hizo especial énfasis en el tema de políticas de información.

11 COLCIENCIAS. "Políticas Nacionales de Información", en Memorias de la 65 Conferencia General de la IFLA. Bangkok: IFLA, 1999. 
(contemplando presupuesto y aspectos logísticos) y, finalmente hace un recorrido por la experiencia de la implementación de políticas de información en otros países de América Latina.

Por su parte, para el año 2001, el Consejo Nacional de Cultura, adscrito al Ministerio de Cultura, presenta el Plan Nacional de Cultura 2001-2010: Hacia una ciudadanía democrática cultural. ${ }^{12}$ En el marco de este Plan se contemplan las bibliotecas públicas como instituciones fundamentales incluidas en las políticas nacionales de información, cultura y democratización del conocimiento.

El recorrido por las iniciativas de formulación de políticas de información permite ver que la presencia social de la biblioteca pública en Colombia ha vivido en los últimos años un cierto auge y, especialmente, un interés dirigido a vincularla con áreas consideradas como más importantes por la opinión pública, como la información, la cultura, la educación, la lectura y la escritura. Tal hecho haría evidente una actitud reduccionista o, al menos, atomizante de la tarea bibliotecaria al no lograr planteársela a la sociedad como un institución compleja en la que las prácticas de informarse, leer y escribir adquieren, sin lugar a dudas, connotaciones especiales en tanto que se ejercen dentro del ámbito de consideraciones y principios que democratizan la producción, circulación y transformación del conocimiento, algo que las bibliotecas públicas han mantenido desde sus mismos inicios. ${ }^{13}$

No obstante, algunas iniciativas sectoriales recientes parecen caminar por senderos más coherentes con esta responsabilidad, en tanto que promueven una presencia activa (más centrada) de la biblioteca pública en los procesos sobre socialización de las personas y consolidación de la democracia. Este es el caso de las iniciativas de la sociedad civil dirigidas a la formulación de una Política Nacional de Lectura y Escritura para Colombia, hechas por ASOLECTURA, en el documento Por una política pública de lectura y escritura: resultados de los Primeros Encuentros Regionales de Lectura y Escritura Colombia 2002, ${ }^{14}$ en el que hace un especial llamado a

Promover la dotación y mejoramiento del sistema de bibliotecas públicas del país, asegurándoles una partida presupuestal anual suficiente para investigación, mantenimiento y (...) desarrollar una estrategia que garantice la presencia de, al

12 Ministerio de Cultura. COnSEJO naCiOnAl De CUltura. Plan Nacional de Cultura 2001 - 2010: Hacia una ciudadanía democrática cultural. Bogotá : Ministerio de Cultura, 2001.

13 Liz Greenhalgh; Ken Worpole y Charles Landry. Libraries in a world of cultural change. Londres : University College London, 1995.

14 ASOlECtURA. Por una política pública de lectura y escritura: resultados de los Primeros Encuentros Regionales de Lectura y Escritura Colombia 2002. Bogotá: ASOLECTURA, ICBF, 2002. $43 \mathrm{p}$. 
menos, una biblioteca pública en cada municipio. Pero una biblioteca en todo el sentido de la palabra, no un remedo de ella. ${ }^{15}$

Y sucede lo mismo con las iniciativas gubernamentales plasmadas en el Documento CONPES 3222: Lineamientos del Plan Nacional de Lectura y Bibliotecas, ${ }^{16}$ publicado en el 2003 y que se constituye en pieza central del Plan Nacional de Desarrollo Hacia un Estado Comunitario, del gobierno de Uribe Vélez (2002-2006) sobre el tema de bibliotecas públicas, lectura, acceso a la información y fomento de la cultura. Este documento es muy explícito al afirmar que:

Las bibliotecas (...) son escenarios fundamentales para el desarrollo de una sociedad. Resultan indispensables en la promoción de la lectura y el mejoramiento de la calidad de la educación al poner al servicio de las comunidades los libros y demás servicios culturales y educativos. ${ }^{17}$

Pasando ya al ámbito local (Departamento de Antioquia) ${ }^{18}$ la revisión de la literatura muestra una serie de acciones estatales relacionadas con la biblioteca pública que se extienden en el tiempo. Se resalta el trabajo realizado en 1972 por la Secretaría de Educación y Cultura de Antioquia para crear la Red Departamental de Bibliotecas Públicas Escolares y la Casa de la Cultura de Antioquia, mediante la Ordenanza $011 ;{ }^{19}$ con la cual se buscaba crear e impulsar los servicios bibliotecarios de la región de una manera equitativa y ordenada.

La Red inició el trabajo con 16 bibliotecas públicas ya existentes en algunos Municipios, y contempló o bien la creación de una biblioteca pública municipal, o la de una biblioteca escolar o una casa de la cultura en cada uno de los 125 municipios antioqueños. Una vez sancionada la Ordenanza 011, la Red orientó y realizó acciones encaminadas a buscar la participación activa de las comunidades para el desarrollo de las bibliotecas; siempre con la perspectiva de integrar los aspectos físicos, económicos y sociales con el fin de racionalizar recursos, aunar esfuerzos y lograr un solo propósito: la creación, consolidación y dinamización de una biblioteca pública en cada uno de los municipios del departamento de Antioquia. Este fin logró materializarse con

15 Ibid.; p. 22

16 CONSEJO NACIONAL DE POLítiCA ECONÓMICA Y SOCIAL. Documento CONPES 3222: Lineamientos del Plan Nacional de Lectura y Bibliotecas. Bogotá: CONPES, 2003.

17 Ibid., p. 3

18 Colombia, administrativamente está dividido en departamentos. El Departamento de Antioquia es el segundo en número de habitantes y en desarrollo económico y social.

19 ANTIOQUIA. ASAMBLEA DEPARTAMENTAL. Ordenanza 11 del 9 de noviembre de 1972. Medellín : Asamblea Departamental, 1979. 
más de 400 bibliotecas. ${ }^{20}$ La principal función de la Red fue la asesoría para la creación, funcionamiento y administración de las bibliotecas públicas, además del suministro de recursos, en especial los de material bibliográfico, con el fin de

Apoyar el desarrollo socio-cultural de la población a través de la organización y dirección de la Red Departamental de Bibliotecas Público-Escolares y Casas de la Cultura. ${ }^{21}$

A partir de la Constitución de 1991 y debido al cambio de las competencias administrativas, los objetivos de la Red fueron posteriormente modificados, pues ya no era de su competencia la dotación bibliográfica de muebles y equipos ni la adaptación de locales, sino que eran competencia del municipio. ${ }^{22} \mathrm{De}$ esta manera el cambio de competencias administrativas en el ámbito nacional genera en las localidades la responsabilidad en las bibliotecas públicas.

\section{Políticas públicas Para LaS BIbliotecas públicas EN UN CONTEXTO LOCAL}

Históricamente Colombia ha sido reconocida como un país de regiones, pero a partir de la promulgación de la Constitución Política de 1991, y con ella de la descentralización del país, se puede hablar de él como un país de localidades (municipios). Tal como lo expresa el artículo 311 de la Constitución:

Al municipio como entidad fundamental de la división político - administrativa de Estado le corresponde prestar los servicios públicos que determine la Ley, construir las obras que demande el progreso local, ordenar el desarrollo de su territorio, promover la participación comunitaria, el mejoramiento social y cultural de sus habitantes y cumplir las demás funciones que le asigne la Constitución y las Leyes. ${ }^{23}$

La descentralización es una apuesta estatal por el desarrollo del país y se centra en las localidades y sus potencialidades de avance regional a través del

20 Orlanda Jaramillo. et al. Historia y desarrollo de la biblioteca pública en Medellín durante el siglo XX: Informe final de investigación. Medellín: Universidad de Antioquia, Escuela Interamericana de Bibliotecología, Centro de Investigaciones en Ciencia de la Información CICINF, 2004. p. 95.

21 Asamblea Departamental. Gaceta Departamental. Año LXXvi No.11.178. Medellín, martes 22 de julio de 1986. p. 1123

22 Un municipio es una unidad territorial administrativa que se conforma con un mínimo de 5.000 habitantes.

23 COlOmbia. COngreso. Constitución Política 1991. Bogotá : El Congreso, 1991. p. 120. 
municipio. Apuesta que se propone que las necesidades y preferencias locales sean expresadas, escuchadas y resueltas desde el nivel de gobierno más cercano a las personas, y que tiene mayor capacidad para hacer diagnósticos y proyectos reales y pertinentes sobre las necesidades de los habitantes, y así responder a ellas de una manera eficiente, eficaz y oportuna.

En este sentido hay que decir que la realidad colombiana presenta un proceso de descentralización aún no consolidado, pues existen razones sociales, económicas y políticas que interfieren en éste, razones especialmente referidas a fenómenos de exclusión social y marginación que todavía se manifiestan en las localidades. Este panorama plantea la necesidad de proponer soluciones a los problemas que tienen que ver con las tensiones existentes entre la municipalidad (lo local) y la educación, la cultura, la participación y la promoción de la ciudadanía.

En este escenario específico, el Estado y la sociedad están llamados a revisar, de manera sistemática, las instituciones sociales que intervienen en estos procesos de ciudadanización, tales como la escuela, las organizaciones civiles y la biblioteca pública, entre otras. En consecuencia se advierte la necesidad de revisar y actualizar las estrategias estatales que están impulsando la construcción de ciudadanía en el marco de un cambio social, cultural y político.

En el contexto para consolidar el proceso de construcción de ciudadanía, la biblioteca pública, como institución social y cultural, ha jugado y juega un papel importante en el país y la región. Muestra de ello es la tímida pero intencionada inserción de esta institución en los planes y programas de gobierno dentro de los ámbitos nacional, regional y local, especialmente desde la década del 50, y que está enmarcada en un proyecto de formación ciudadana mediante el fomento de la educación y la formación cívica de valores que contienen la identidad nacional.

Igualmente, desde finales de los años 60, el Instituto Colombiano de Cultura - COLCULTURA - y más recientemente el Ministerio de Cultura, se han encargado de dinamizar planes y programas de carácter estatal y gubernamental para desarrollar la biblioteca pública como un instrumento de participación e inserción de las personas en la vida política del país y de las regiones.

\section{CONSIDERACIONES METODOLÓGICAS}

SOBRE EL DISEÑO E IMPLEMENTACIÓN DE LAS POLÍTICAS

Ya planteado lo anterior se hace necesario proponer algunas ideas en torno al diseño e implementación de las políticas públicas para bibliotecas públicas en Colombia, y así garantizar el fomento de la acción política y el estímulo de 
la diversidad de intereses y pensamientos en los ámbitos nacional, regional y local.

Una política pública para la biblioteca pública debe propender hacia el ejercicio y el cumplimiento del derecho a la información, a la educación y a la cultura de un conglomerado social; además de considerar a la biblioteca pública como, "fuerza viva al servicio de la enseñanza, la cultura y la información, indispensable para el fomento de la paz y de la comprensión entre las personas y las naciones". ${ }^{24}$ En tal sentido, la política pública debe encaminarse hacia la promoción de las demandas y transformaciones sociales relacionadas con la circulación de la información y la producción de conocimiento en las comunidades, promoviendo la representatividad social de la biblioteca pública; y hacia la cualificación de las iniciativas estatales dirigidas a formular legislación que respalde a esta institución social. De esta manera, la política pública contribuiría a configurar la agenda pública dentro de la cual sea posible desarrollar las transformaciones sociales y políticas que el país requiere, así como específicamente provocar la alineación de las posiciones y fuerzas (sociales y estatales) que puedan integrar los planes, programas, proyectos y acciones encaminadas a permitir el acceso a la cultura y la educación.

Lo anterior estaría en consonancia con lo establecido en la Constitución Política de Colombia (1991), que le exige al Estado Colombiano diseñar y poner en marcha iniciativas que promuevan el desarrollo de la biblioteca pública, una de las principales instituciones que garantizan el derecho a la información, y el acceso a la cultura, a la educación y a la recreación; derechos estipulados en nuestra Carta Magna.

De igual forma, el plan de desarrollo de cada administración municipal se constituye en un marco para la consolidación de este propósito: centrar la atención en el diseño y la ejecución de acciones que posibiliten potenciar y redimensionar el papel sociocultural que juega la biblioteca pública, expresado en su rentabilidad social, y en el acceso a la información, al conocimiento, a la educación y a la cultura.

Desde un enfoque interpretativo y teniendo en cuenta que en la formulación, desarrollo y aplicación de una política pública se deben conjugar tanto las acciones estatales (políticas, legislación, etcétera), como las iniciativas socioculturales de la comunidad, se hace necesario iniciar una revisión de la legislación relacionada con la biblioteca pública como objeto de una política pública, pues en la mayoría de los países de la región esta legislación está inmersa en disposiciones culturales y educativas. 
Con lo anterior se busca identificar la visión que el Estado tiene sobre la biblioteca pública como institución social que intenta construir la ciudadanía y el desarrollo local. También es preciso compilar y revisar las acciones sociales relacionadas con la promoción de la biblioteca pública como centro de desarrollo local y los avances de instituciones, grupos y gremios en la construcción de planes, programas o proyectos relacionados con esta institución.

En la lectura de la legislación se hace necesario poner las disposiciones legales en sus contextos respectivos, con el objeto de entender y comprender tanto a sus autores, como sus contenidos y a sus destinatarios, lo cual se puede hacer con base en una perspectiva hermenéutica. Lo anterior no podrá desconocer el desarrollo histórico de la legislación cultural y educativa del país, y de esta manera la comprensión de la legislación se relacionará con los aspectos semánticos (conexión entre las disposiciones legales y los aspectos que éstas cubren) y con aspectos pragmáticos (contextos de aplicación de las disposiciones y su reconocimiento social).

Por otra parte, el análisis textual se constituye como una técnica que permite resolver las necesidades metodológicas asociadas con la tarea de relacionar la legislación y las iniciativas sociales a partir de categorías básicas de análisis asociadas con la relación Estado - Sociedad - Biblioteca. Para ello es necesario antes establecer y definir un sistema de categorías que explique las relaciones fundamentales entre biblioteca pública - educación; biblioteca pública - cultura, y biblioteca pública - participación, así como otras que puedan emerger de la interpretación hermenéutica de la legislación y de las propuestas sociales.

La revisión de las acciones estatales y socioculturales y de las relaciones establecidas entre ellas, permitirá conocer la visibilidad de la biblioteca pública, y así proponer la integración de las acciones políticas impulsadas por el Estado y las acciones socioculturales impulsadas por la comunidad. Integración que habrá de responder a la necesidad de formular estrategias "de orden político, sociocultural y gerencial por medio de las cuales sea posible formular y llevar a cabo la política pública" 25 y que se constituye en la base para configurar propuestas de política pública.

\section{A MODO DE CONCLUSIONES}

En un contexto de descentralización administrativa se hace necesario plantear el desarrollo de políticas públicas en el ámbito de las localidades; políticas que 
deberán responder al posicionamiento y la visibilización de la biblioteca pública, y contribuir al desarrollo nacional.

En esta misma perspectiva, las políticas públicas para el desarrollo local deben ser el resultado de una revisión sistemática de las instituciones sociales que intervienen en los procesos de ciudadanización de las personas, tales como la escuela, las organizaciones civiles o la biblioteca pública. En consecuencia se advierte la necesidad de revisar y actualizar las estrategias estatales que están impulsando la construcción de ciudadanía, dentro del marco de un cambio social, cultural y político a través de la biblioteca pública.

Finalmente, ni el país ni las localidades colombianas han contado con una verdadera política para el desarrollo de las bibliotecas públicas, lo que ha existido son diversos y discontinuos intentos que no han llegado a consolidarse; de ahí la necesidad de formular una política pública para las bibliotecas públicas, que permita conjugar las acciones estatales, privadas y sociales, y de esta manera poder articular planes, programas, proyectos y procesos encaminados al desarrollo de la biblioteca pública y a su inserción en la vida política de las personas. En otras palabras, es urgente y necesario encaminar las acciones estatales y socioculturales desarrolladas en el país y las localidades a favor de las bibliotecas públicas y el acceso social a la información, de manera que se logren articular y generar espacios de participación y de acceso democrático a los bienes y servicios culturales, educativos, informativos y recreativos, a los que todos los ciudadanos tienen derecho.

\section{BiBLIOGRAFÍA}

Agudo, Álvaro. "Relaciones posibles entre la biblioteca pública y su comunidad", en Bibliotecas públicas y escolares. Medellín: FUNDALECTURA, 2001. pp. 21-26.

Arango Velásquez, Gabriel Jaime. "Valor y función de la información en los procesos comunitarios y ciudadanos de construcción de identidades culturales", en Revista Interamericana de Bibliotecología. Medellín. Vol. 24, No. 2 (Jul. - Dic., 2001); pp. 83-99

ASOLECTURA. Por una política pública de lectura y escritura: resultados de los Primeros Encuentros Regionales de Lectura y Escritura Colombia 2002. Bogotá: ASOLECTURA, ICBF 2002. 43 p.

Barbero, Jesús Martín. "Dinámicas urbanas de la cultura”, en Revista Gaceta de Colcultura. No. 12 (1991).

Becerra H, Luis Alberto. La biblioteca pública como centro de desarrollo cultural. Cali: Biblioteca Nacional de Colombia, 1992. 68 p. 
Betancur Betancur, Adriana María y Álvarez Zapata, Didier. "Servicios de información ciudadanos y comunitarios: nuevos sentidos para las bibliotecas públicas", en Revista Interamericana de Bibliotecología. Medellín. Vol. 24, No. 2 (Jul. - Dic., 2001); pp. 13-24

"La biblioteca pública y su desafío frente a la globalización”, en Bibliotecas públicas y escolares. Medellín: FUNDALECTURA, 2001. pp. 10-20

Castrillón Zapata Silvia. “¿Crea la biblioteca ciudadanos mejor informados?”, en Revista Interamericana de Bibliotecología. Medellín. Vol. 24, No. 2 (Jul. - Dic., 2001); pp. 103-111

CERLALC. Las políticas nacionales de lectura. [Bogotá]: CERLALC, 1999. pp. $37-40$

COLCIENCIAS. "Políticas Nacionales de Información”, en Memorias de la 65 Conferencia General de la IFLA. Bangkok: IFLA, 1999

. Plan estratégico de los sistemas de información científica y tecnológica. Bogotá: COLCIENCIAS, 1995.60 p.

COlOmbia. COngreso. Constitución Política de Colombia. Bogotá : El Congreso, 1991.276 p.

COLOMBIA. MINISTERIO DE CULTURA. Plan Nacional de cultura 2001 2010: bacia una ciudadanía democrática cultural. Bogotá : Ministerio de Cultura, 2001.56 h.

CONSEJO NACIONAL DE POLÍTICA ECONÓMICA Y SOCIAL CONPES. Documento CONPES 3222: Lineamientos del Plan Nacional de Lectura y Bibliotecas. Bogotá : El Consejo, 2003

Cosials, Alex. "Biblioteca publica y cohesión social", en Educación y Biblioteca. Madrid. Vol. 15, No. 136 (Jul. - Ago. 2003); pp. 88-92

Dávila L. Andrés. "Legitimidad y orden político: consideraciones sobre su interrelación en la sociedad contemporánea”, en Estudios Políticos. Medellín. No. 7 - 8 (Dic. 1995 - Jun. 1996); pp. 10-23

Delors, Jacques. La educación encierra un tesoro. México: UNESCO, 1997. 318 p.

Greenhalgh; Liz, Worpole, Ken y Landry, Charles. Libraries in a world of cultural change. Londres: University College London, 1995

Gosepath, Stefan. "Consideraciones sobre las fundamentaciones de los derechos humanos sociales", en Ciudadanía y derechos bumanos sociales. Medellín : Ediciones ENS, 2001

IFLA. Servicio de bibliotecas públicas: directrices IFLA/UNESCO para el desarrollo. Bogotá: FUNDALECTURA, 2002. 122 p.

Ministerio DE CUltura. CONSEJO NACiONAl DE CUltura. Plan Nacional de Cultura 2001 - 2010: Hacia una ciudadanía democrática cultural. Bogotá: Ministerio de Cultura, 2001.

Morales Campos, Estela. El derecho a la información y las políticas de información en América Latina. Ponencia presentada en la 65 Conferencia General de la IFLA. Agosto de 1999. 14 p. 
Petit, Michéle. “¿Cómo pueden contribuir las bibliotecas y la lectura a luchar contra la exclusión?” en Bibliotecas públicas y escolares. Medellín: FUNDALECTURA, 2001. pp. 66-87

POLÍTICAS NACIONALES DE INFORMACIÓN, en Memorias de la 16 Conferencia General de la IFLA. Bangkok: IFLA, 1999

Rodas Cortés, Francisco. De la política de la libertad a la política de la igualdad. Medellín : Siglo del Hombre Editores : Universidad de Antioquia, 1999. 200 p.

"Los derechos humanos sociales: consideraciones sobre su fundamentación a la luz del liberalismo y del igualitarismo", en Estudios Políticos. No. 15 (Dic. 1999); pp. 109-126

REUNIÓN INTERNACIONAL SOBRE FORMULACIÓN DE POLÍTICAS NACIONALES DE LECTURA (1 : Caracas : 1992). Memorias, en FUNDALECTURA. Hojas de Lectura. Bogotá: FUnDALECTURA. No. 19-20 (Dic. 1992 - Feb. 1993); pp. 4-6

REUNIÓN REGIONAL SOBRE EL ESTADO ACTUAL Y LAS ESTRATEGIAS PARA EL DESARROLLO DE LOS SERVICIOS DE BIBLIOTECAS PÚBLICAS EN AMÉRICA LATINA Y EL CARIBE (1982 : Caracas). Declaración de Caracas sobre la biblioteca pública como factor de desarrollo e instrumento de cambio social en América Latina y el Caribe: informe final. Caracas : PGI. UNISIST, 1982. 23 p.

Revesz, Bruno. "Políticas públicas en América Latina y el papel estratégico de las bibliotecas públicas: nuevos desafíos", en Reunión Regional de Bibliotecas Públicas (1 : 1996 : Piura, Perú). Ponencia de la Reunión Regional de Bibliotecas Públicas. Piura: IFLA, 1996. $15 \mathrm{~h}$.

"Políticas de información, bibliotecas públicas y desarrollo local y regional", en Revista Interamericana de Bibliotecología. Medellín. Vol. 24, No. 2 (Jul. - Dic., 2001); pp. 45-63

Rodríguez, Pedro Gerardo. "Políticas Nacionales de Lectura", en De Antología. Bogotá. No. 1 (2002); pp. 104-112

Sáenz Vargas, Carlos Roberto (Comp.). Documentos críticos sobre legislación cultural. Bogotá. Instituto Colombiano de Cultura COLCULTURA, $1993.190 \mathrm{p}$.

Suaiden, Emir José. Biblioteca publica e Informaçao a comunidade. Brasil: Global Medica, 1995. 185 p.

UNESCO. "Manifiesto de la Unesco sobre la biblioteca pública 1994", en Hojas de Lectura. Bogotá. No. 35 (Ago. 1995); pp. 4-5

Villegas Puerta, Luis Eduardo. Anotaciones sobre políticas públicas de lectura y escritura. (Mimeo). Medellín: s.n, 2002. 4 h. 